\title{
Demographic Risk Factors of Retinopathy of Prematurity: A Systematic Review of Population- Based Studies
}

\author{
Caberry W. Yu ${ }^{a}$ Marko M. Popovic ${ }^{b}$ Arjan S. Dhoot ${ }^{c}$ Parnian Arjmand ${ }^{b}$ \\ Rajeev H. Muni ${ }^{\text {b, d, e }}$ Nasrin N. Tehrani ${ }^{\text {b, e }}$ Kamiar Mireskandarib, e \\ Peter J. Kertes ${ }^{b, e, f}$ \\ aDepartment of Surgery, McMaster University, Hamilton, ON, Canada; ${ }^{b}$ Department of Ophthalmology and Vision \\ Sciences, University of Toronto, Toronto, ON, Canada; 'Faculty of Medicine, University of Toronto, Toronto, ON, \\ Canada; 'Department of Ophthalmology, St. Michael's Hospital/Unity Health Toronto, Toronto, ON, Canada; \\ eDepartment of Ophthalmology and Vision Sciences, The Hospital for Sick Children, Toronto, ON, Canada; ${ }^{\mathrm{f} J o h n}$ and \\ Liz Tory Eye Centre, Sunnybrook Health Sciences Centre, Toronto, ON, Canada
}

\section{Keywords}

Retinopathy of prematurity - Gestational age - Birth weight · Neonatal screening P Population

\begin{abstract}
Introduction: Current national guidelines use gestational age (GA) and birth weight (BW) as their basis for retinopathy of prematurity (ROP) screening. The strength of association of these and other demographic risk factors is inconsistent across studies. This review aims to evaluate the strength of association of documented risk factors for ROP in large sample, population-based studies. Methods: MEDLINE, EMBASE, and Cochrane Library were searched from January 2010 to May 2020. Original studies reporting the risk of ROP in a region and demographic risk factors were included. Results: Eighteen studies comprising 342,005 infants were included. The overall risk of ROP in preterm infants was $18.8 \%$. For every week decrease in GA, there was a median adjusted odds ratio (aOR) of 1.4 times (range 1.2-1.9) of developing ROP. For every 100-g decrease in BW, the median aOR was 1.8 times (range 1.2-2.7). Higher risk was found in infants with
\end{abstract}

Karger@karger.com www.karger.com/neo

Karger $\stackrel{\text { ' }}{=}$

BOPEN ACCESS
(C) 2022 The Author(s)

Published by S. Karger AG, Basel

This is an Open Access article licensed under the Creative Commons Attribution-NonCommercial-4.0 International License (CC BY-NC) (http://www.karger.com/Services/OpenAccessLicense), applicable to the online version of the article only. Usage and distribution for commercial purposes requires written permission. neonatal sepsis and bronchopulmonary dysplasia. The risk of any, severe, and treatment-requiring ROP was highest for 23 weeks GA, which was $66.5,40.3$, and $39.4 \%$, respectively. Regions with higher neonatal mortality rates had the highest mean GA of infants with ROP. Conclusion: For every week decrease in GA and every 100-g decrease in BW, there was a median of 1.4 times and 1.8 times the odds of developing ROP, respectively. Further research is required to clarify the role of additional risk factors.

(C) 2022 The Author(s). Published by S. Karger AG, Basel

\section{Introduction}

Retinopathy of prematurity (ROP) is a retinal vasoproliferative disease that occurs in preterm infants. It is a leading cause of visual impairment and blindness in children [1]. The development of retinal vasculature starts at 16 weeks of gestation and concludes at term [2]. It is well known that ROP begins with the growth arrest of retinal vessels in premature babies exposed to high levels of oxygen at birth. The resulting hypoxia leads to
Correspondence to:

Caberry W. Yu, wyu@ qmed.ca

Peter J. Kertes, peter.kertes@ sunnybrook.ca 
the upregulation of angiogenic growth factors such as vascular endothelial growth factor (VEGF) and the risk of the abnormal neovascularization that may lead to irreversible vision loss [2]. In the subsequent phase, hypoxia-induced angiogenic factors are produced, driving compensatory mechanisms that result in aberrant retinal vascularization [2]. When an infant is born prematurely, vascular immaturity predisposes the retina to complications such as ROP that may lead to irreversible vision loss $[2,3]$. The number of infants at risk of ROP is expected to increase as survival of preterm infants improve $[4,5]$.

Current national ROP guidelines use the gestational age (GA) and birth weight (BW) as their basis for screening. In the USA, the American Academies of Ophthalmology and Pediatrics created a joint guideline that recommends all infants $\leq 30$ weeks of GA or $\leq 1,500 \mathrm{~g}$ of BW be screened for ROP, as well as other infants deemed at risk based on clinical course [6]. The criteria for screening guidelines around the world range from $<30$ to 37 weeks of GA and from $<1,000$ to $2,500 \mathrm{~g}$ of BW [7]. The oldest babies routinely screened were $<37$ weeks $G A$ and $<2,000$ g BW in a 2018 study in Egypt and Ukraine [7]. The strength of association between major demographic risk factors and the development of ROP has been examined in CRYO-ROP study and multiple subsequent studies with varying associations [8-12]. To help inform risk assessment, this review was conducted to provide a more precise estimation of the association between risk factors and ROP at a population level.

\section{Methods}

\section{Search Methods}

This systematic review was registered in the International Prospective Register of Systematic Reviews database (CRD42020185343). A systematic literature search was conducted on Ovid MEDLINE, EMBASE, and Cochrane Library (January 1, 2010, to May 9, 2020; online suppl. Table 1; for all online suppl. material, see www.karger.com/doi/10.1159/000519635). Studies published prior to 2010 were excluded to reflect the latest advances in neonatal care. Reference lists of included articles were also searched manually to elicit further relevant literature.

\section{Eligibility Criteria}

The following inclusion criteria were used in the selection of studies: (1) original, population-based studies that reported on the risk of ROP for an eligible cohort of a geographically defined region (e.g., city, state, province, country, etc.), and (2) studies that investigated demographic risk factors of the mother or infant. Exclusion criteria included studies that were not peer-reviewed, forthcoming, or available in English, as well as any that only re- ported on the risk of a single subtype of ROP (e.g., treated ROP). When articles contained repeat data from another study, the larger dataset was taken.

\section{Study Selection}

Studies were selected via an independent two-stage screening process by two authors (C.W.Y., A.S.D.), first by screening of titles and abstracts, followed by full-text screening. Microsoft Excel (Microsoft Corporation, Redmond, WA, USA) was used to manage the identified records and eligibility status. The systematic review adhered to the tenets of the Declaration of Helsinki.

\section{Data Collection and Risk of Bias Assessment}

For all included studies, the following parameters were considered: identifying data (e.g., authors, title, country of publication), study design, screening guidelines for ROP in the region, demographic characteristics (e.g., GA, BW, maternal age), systemic complications (e.g., pneumonia, mortality), and ROP characteristics (e.g., stage, treatment). For all studies, severe ROP was defined as ROP stage 3 or worse in at least one eye, and treatment-requiring ROP was defined as ROP treated with laser, cryotherapy, anti-VEGF injections, or surgery. Countries were classified by neonatal mortality into region 1 or region 2 , whereby the former represents countries with neonatal mortality $<5$ per 1,000 live births in 2019 [13]. Only premature infants ( $\mathrm{GA}<37$ weeks) were included in the data analysis.

The primary outcome was the strength of association of demographic risk factors with ROP risk. Secondary outcomes included the regional risk of any, severe, and treatment-requiring ROP stratified by GA and BW. The associations between additional demographic risk factors and ROP were also collected. Microsoft Excel (Microsoft Corporation, Redmond, WA, USA) was used throughout the data collection process, which was performed independently by two authors (C.W.Y. and A.S.D.). Conflicts in screening and data collection were resolved via consultation of a third reviewer (M.M.P.).

Risk of bias was assessed using a validated tool for prevalence studies published by Hoy et al. [14]. It consists of ten items addressing four domains of bias: selection, nonresponse, measurement, and analysis. Each domain was scored by “yes" (zero points), "no" (one point), or "not reported" (one point). A summary assessment ranked each study into low (zero to three points), moderate (four to six points), or high (seven to nine points) risk of bias [14]. Quality of evidence for the main outcomes was assessed using Grading of Recommendations, Assessment, Development and Evaluation (GRADE) recommendations, whereby evidence was graded by five criteria: risk of bias, inconsistency, indirectness, imprecision, and publication bias $[15,16]$. Assessments were performed independently by two authors (C.W.Y. and A.S.D.).

\section{Data Synthesis and Analysis}

Throughout, continuous data were collected with means \pm standard deviations, whereas categorical parameters were collected using percentages of the total sample. In the reporting of the pooled risk of all outcomes, the weighted mean was used to report overall risk. It was defined as:

$$
\bar{X}=\frac{\sum_{i=1}^{n} w_{i} x_{i}}{\sum_{i=1}^{n} w_{i}}
$$

with the number of infants representing the weighting factor. Odds ratios were also collected and reported based on the individual studies. 


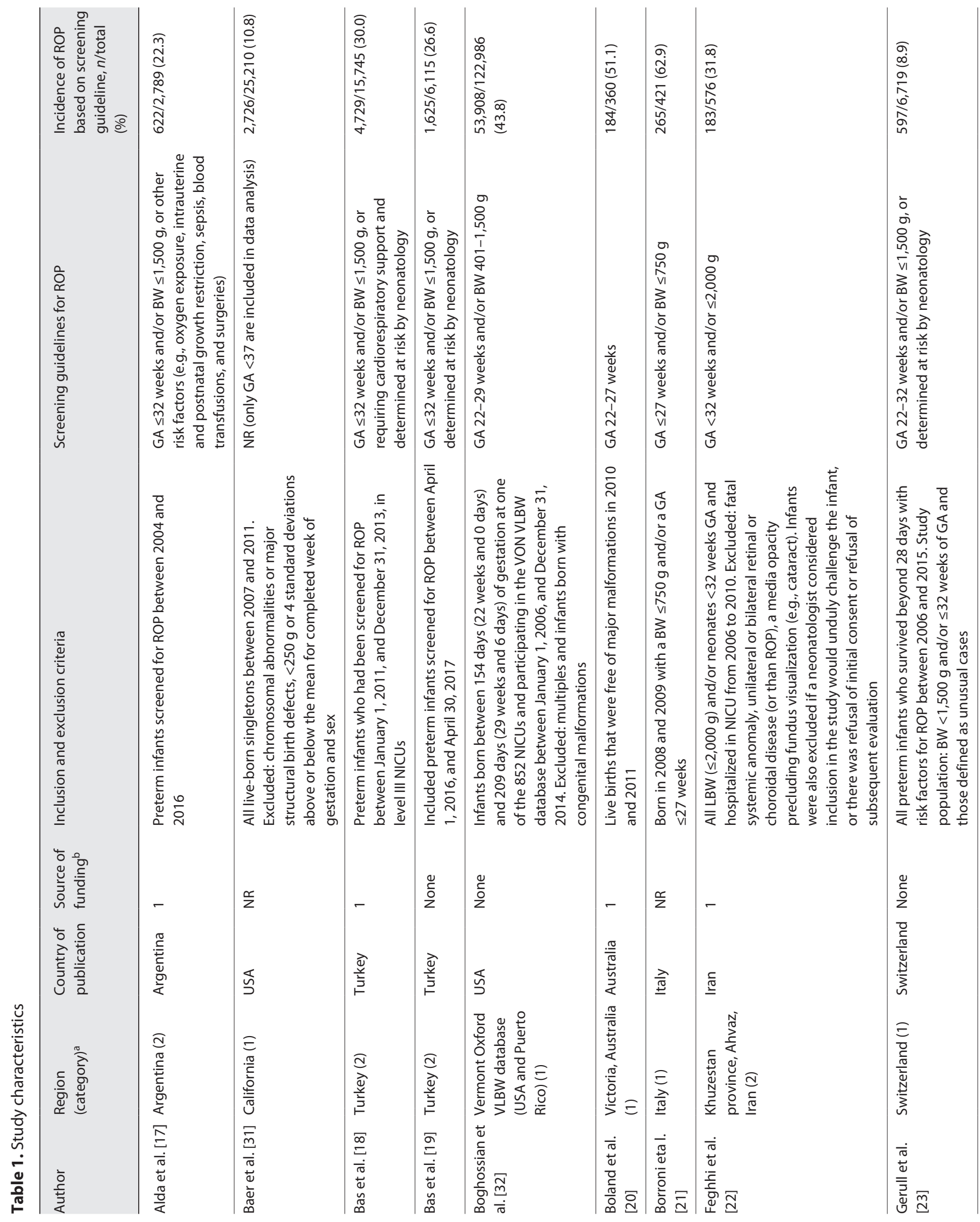




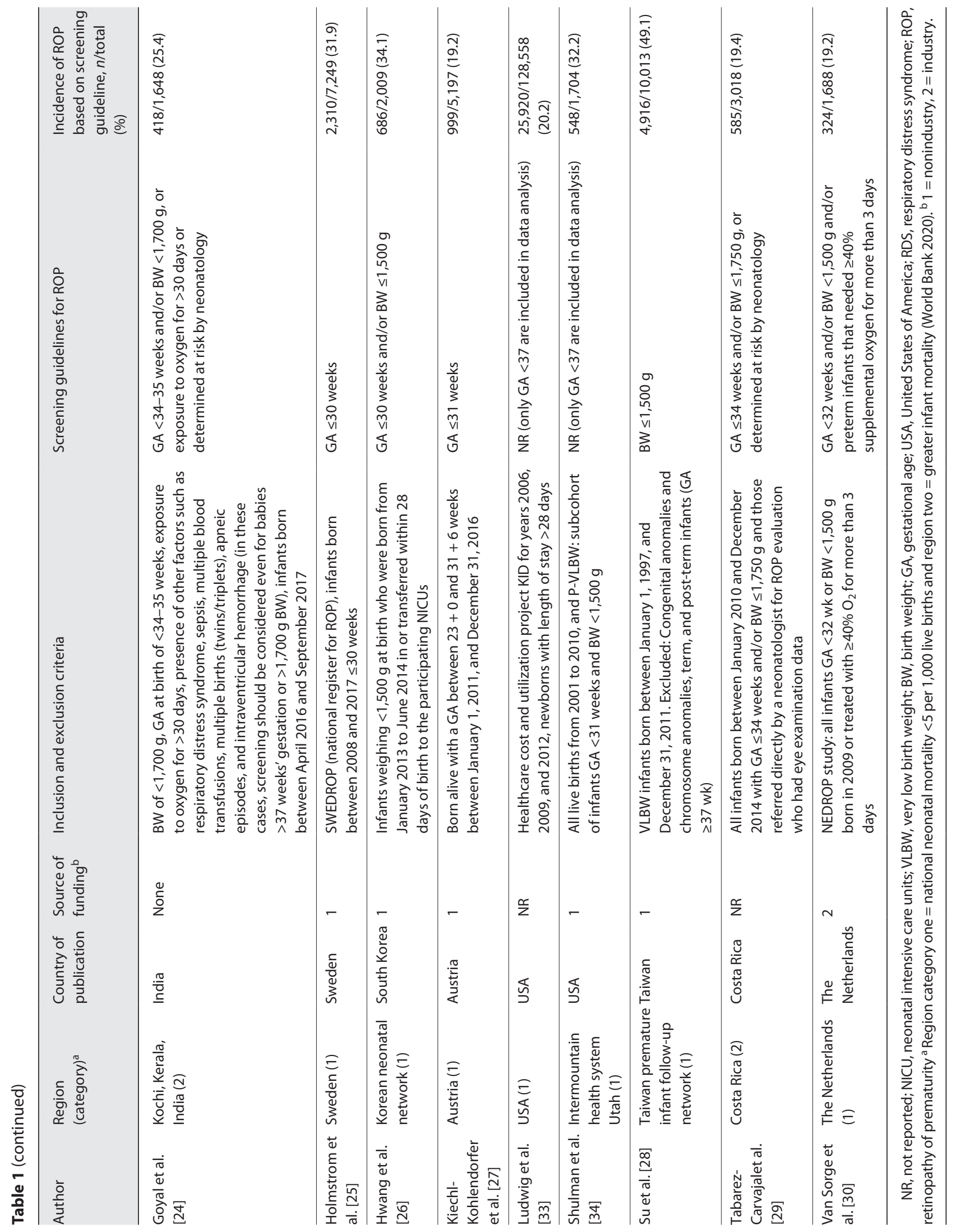




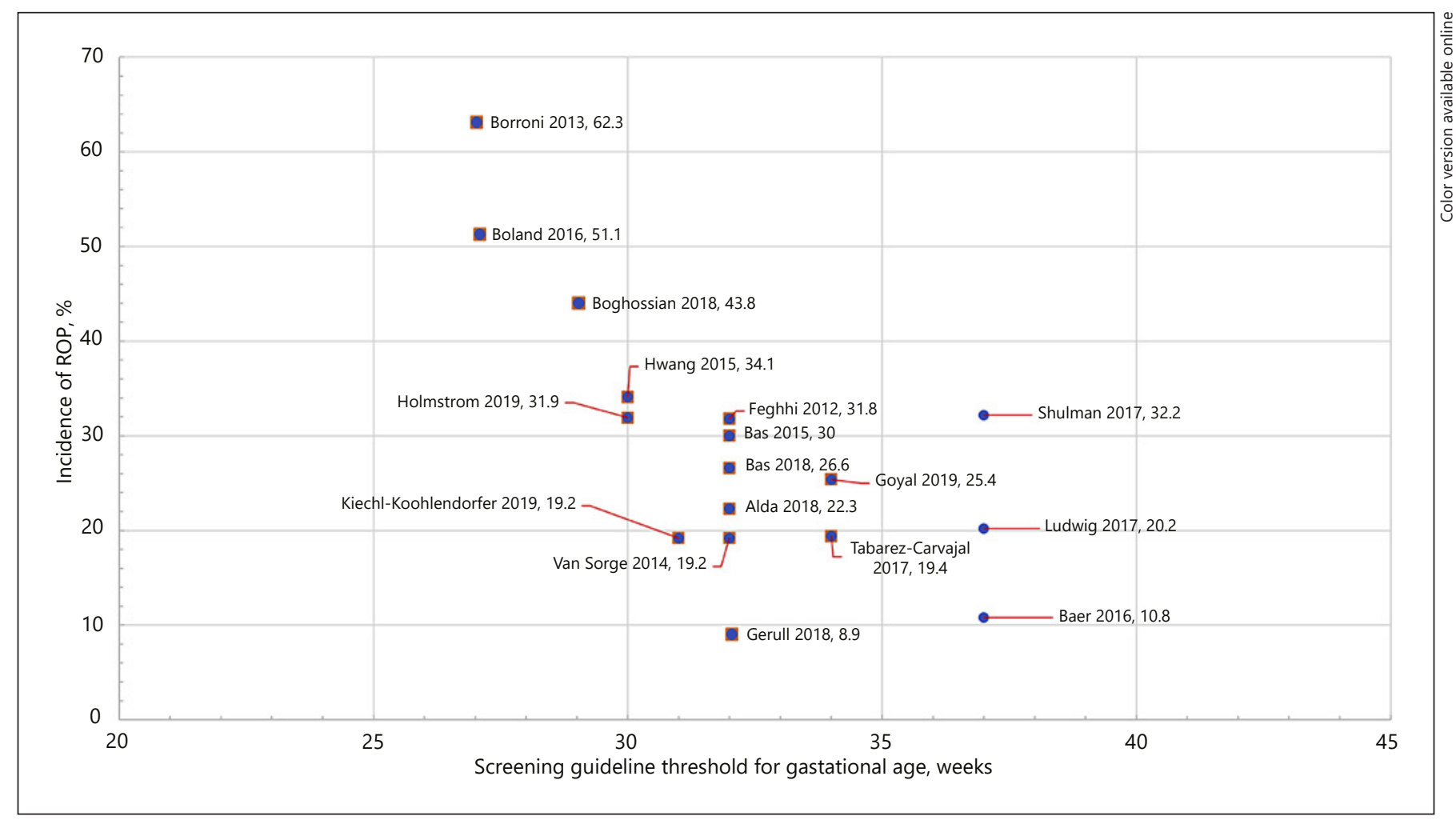

Fig. 1. Incidence of ROP by screening guideline upper limit of GA.

Pooled estimates of ROP risk were provided only if two or more studies were included for the same comparator outcome. For associations between demographic characteristics and ROP, a $p$ value of less than 0.05 was determined a priori to denote statistical significance. No meta-analysis was performed.

\section{Results}

\section{Study Characteristics}

In total, 4,091 results were identified in the database search, of which 104 articles proceeded to full-text screening (online suppl. Fig. 1). Eighteen studies $(n=342,005)$ were included [17-34]. A complete listing of baseline demographic information for each included study can be found on Table 1.

Studies investigated regions of 14 countries or autonomous states: the USA $(n=4)$, Turkey $(n=2)$, Argentina $(n=1)$, Australia $(n=1)$, Austria $(n=1)$, Costa Rica $(n=$ $1)$, India $(n=1)$, Iran $(n=1)$, Italy $(n=1)$, The Netherlands $(n=1)$, Sweden $(n=1)$, Switzerland $(n=1)$, South Korea $(n=1)$, and Taiwan $(n=1)$. Twelve studies $(n=$ $312,114)$ were performed in geographic region 1 (i.e.,
$<5 / 1,000$ neonatal mortality), while six studies ( $n=$ 29,891 ) were performed in geographic region 2 (i.e., $>5 / 1,000$ neonatal mortality). Overall, 7 studies were prospective and 11 studies were retrospective.

\section{Patient Characteristics}

Online supplementary Table 2 outlines patient characteristics. The mean GA of each study ranged from 25.6 to 31.7 weeks, and the mean BW of each study ranged from 812.0 to $1,468.4 \mathrm{~g}$. The weighted mean sex distribution was $52.3 \%$ male (range $48.3-63.7 \%$ male). Nine studies reported on the risk of other neonatal complications (online suppl. Table 2). The most commonly reported complications were bronchopulmonary dysplasia/chronic lung disease (BPD/ CLD, $n=6)$, intraventricular hemorrhage $(n=6)$, necrotizing enterocolitis $(n=6)$, and neonatal sepsis $(n=5)$.

\section{Risk of Bias Assessment and GRADE Certainty of Evidence}

All 18 studies had a high external and internal validity on risk of bias assessment (online suppl. Table 3). All studies scored between zero to three points, suggesting a low overall risk of bias. Most (12/18) studies investigated 


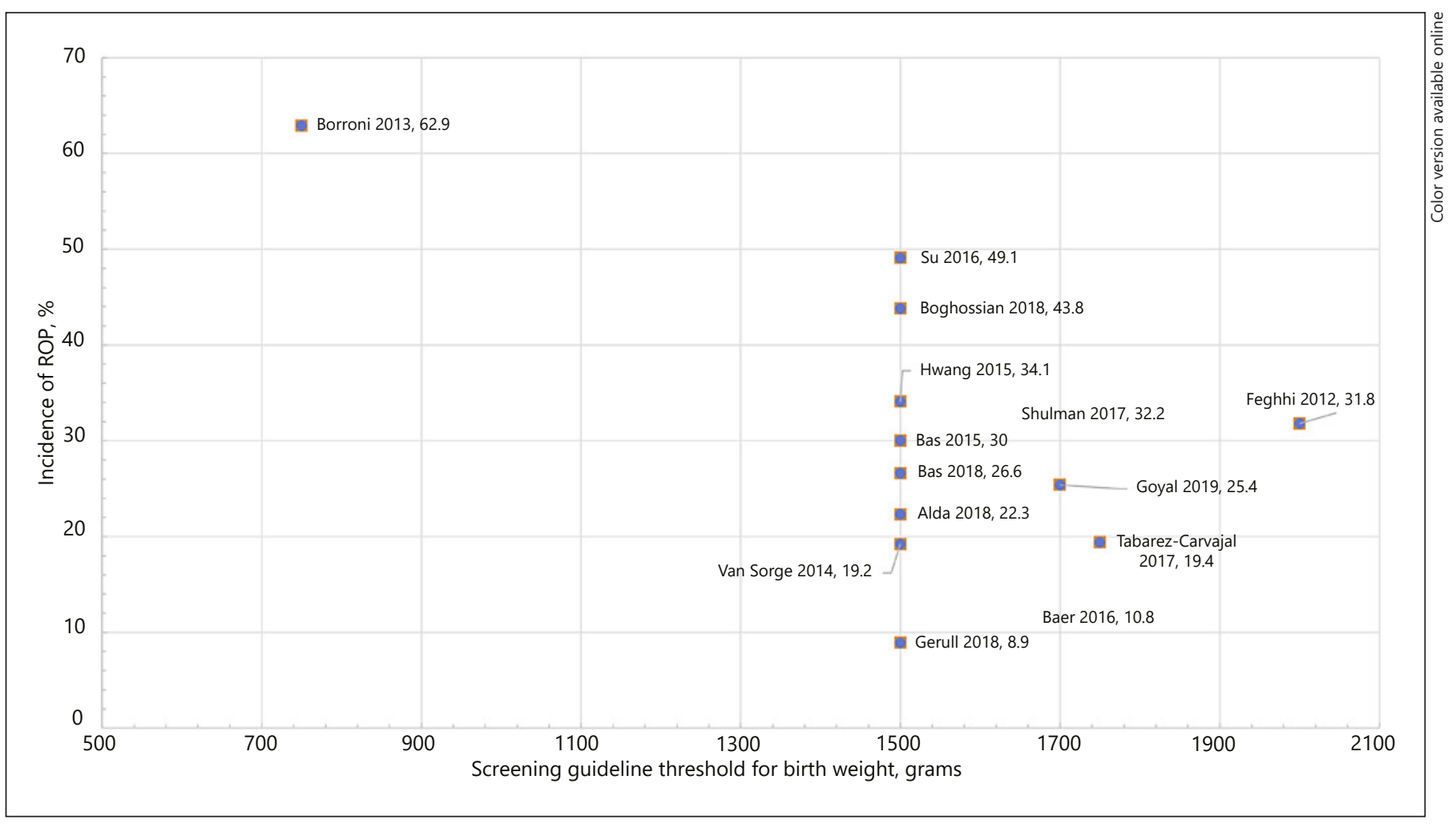

Fig. 2. Incidence of ROP by screening guideline upper limit of BW.

risk at a national level, and all studies had screened all eligible infants or performed random sampling. All studies had appropriate ROP definitions and performed direct and consistent data collection. The main limitation was that the representativeness was limited as only five studies collected from $\geq 75 \%$ of eligible participants of the region. Nine studies did not report the percentage of the target hospital sites or population screened. GRADE certainty of evidence was moderate for almost all examined pooled outcomes due to a low risk of bias, large sample size, and low degrees of inconsistency, indirectness, and imprecision (online suppl. Table 4). Studies either had no funding $(n=4)$, funding by nonindustry sponsors $(n=9)$, funding by both industry and nonindustry sponsors $(n=$ $1)$, or did not report funding details $(n=4)$.

\section{Overview of Screening Guidelines and Risk of ROP}

The screening guidelines for ROP differed between most studies, with the threshold GA ranging from $\leq 27$ weeks to $\leq 34$ weeks $\mathrm{GA}$, and $\mathrm{BW}$ ranging from $\leq 750 \mathrm{~g}$ to $\leq 1,750 \mathrm{~g} \mathrm{BW}$. Three studies did not specify GA and BW thresholds, and only preterm infants ( $<37$ weeks GA) were included in this analysis. The most common screen- ing guideline $(n=5)$ was for all infants in the region born at $\leq 32$ weeks GA and/or $\leq 1,500$ g BW (Table 1) [17-19, $23,30]$. Risk of ROP was highest in the study that screened infants $\leq 27$ weeks GA and/or $\leq 750 \mathrm{~g} \mathrm{BW} \mathrm{(62.9 \% ),} \mathrm{which}$ was the most restrictive threshold of all studies [21]. The incidence of ROP according to each screening criterion is listed in Table 1. Figures 1 and 2 show the incidence of ROP based on GA and BW, respectively.

Pooled data showed that, when examining all preterm infants unrestricted by BW, the overall risk of ROP among 155,472 infants was $18.8 \%$ (range 10.8 to $32.2 \%$ ) [31, 33, 34]. The risk of ROP by zone, stage, and type of treatment are reported in online supplementary Table 5. Severe ROP was investigated in 13 studies ( $n=71,828$ ROP infants). Severe ROP accounted for 20.5\% $(14,744 / 71,828)$ of all ROP cases. The risk of ROP treatment was investigated among 65,631 infants with any ROP. Laser, cryotherapy, anti-VEGF injections, or surgery were performed in $14.8 \%(9,709 / 65,631)$ of all ROP cases.

\section{Primary Analysis: Risk of ROP by $G A$ and $B W$}

The risk of ROP by GA and BW can be found on online supplementary Table 6 . The pooled risk of ROP among 
Table 2. Association between GA or BW and development of ROP

\begin{tabular}{|c|c|c|c|c|c|c|}
\hline Author & \multicolumn{3}{|l|}{ Associations with GA risk factors } & \multicolumn{3}{|l|}{ Associations with BW risk factors } \\
\hline Baer et al. [31] & NR & & & & & \\
\hline Bas et al. [18] & NR & & & & & \\
\hline Bas et al. [19] & GA (weeks) ${ }^{\mathrm{a}}$ & aOR: $0.812^{b}$ & $0.726-0.910$ & $B W(g)^{a}$ & aOR: $0.998^{b}$ & $0.997-0.999$ \\
\hline Borroni et al. [21] & $\mathrm{GA} \leq 24$ weeks & OR: 2.01 & $p=0.008$ & $\mathrm{BW} \leq 750 \mathrm{~g}$ & OR: 1.73 & $p=0.007$ \\
\hline Feghhi et al. [22] & GA (weeks) & OR: 0.52 & $0.41-0.66$ & BW (g) & OR: 0.99 & $0.99-0.997$ \\
\hline Gerull et al. [23] & NR & & & & & \\
\hline Goyal et al. [24] & NR & & & & & \\
\hline \multirow[t]{2}{*}{ Hwang et al. [26] } & GA (weeks) & OR: 0.704 & $0.645-0.767$ & BW (g) with stage $\geq 3$ ROP & OR: 1.001 & $1.000-1.002$ \\
\hline & GA (weeks) with stage $\geq 3$ ROP & OR: 1.017 & $1.000-1.034$ & & & \\
\hline \multirow{6}{*}{ Ludwig et al. [33] } & GA 25-26 weeks (ref: >36 weeks) & aOR: $18.52^{c}$ & $10.46-32.8$ & BW 750-999 g (ref: >2,500 g) & aOR: $4.08^{c}$ & $2.89-5.78$ \\
\hline & GA $27-28$ weeks (ref: $>36$ weeks) & aOR: $16.43^{\mathrm{C}}$ & $9.23-29.27$ & BW $1,000-1,249 \mathrm{~g}($ ref: $>2,500 \mathrm{~g})$ & aOR: $3.60^{c}$ & $2.55-5.09$ \\
\hline & GA $29-30$ weeks (ref: >36 weeks) & aOR: $12.59^{c}$ & $7.05-22.5$ & BW $1,250-1,499 \mathrm{~g}$ (ref: $>2,500 \mathrm{~g})$ & aOR: $3.14^{c}$ & $2.22-4.43$ \\
\hline & GA 31-32 weeks (ref: >36 weeks) & aOR: $8.74^{\mathrm{c}}$ & $4.91-15.55$ & BW $1,500-1,749 \mathrm{~g}$ (ref: >2500g) & aOR: $1.98^{c}$ & $1.41-2.80$ \\
\hline & GA 33-34 weeks (ref: >36 weeks) & aOR: $3.26^{c}$ & $1.84-5.78$ & BW $1750-1,999 \mathrm{~g}$ & aOR: $1.32^{c}$ & $0.93-1.87$ \\
\hline & GA 35-36 weeks (ref: >36 weeks) & aOR: $2.47^{\mathrm{c}}$ & $1.32-4.61$ & BW 2,000-2,499g & aOR: $0.79^{c}$ & $0.56-1.10$ \\
\hline Su et al. [28] & NR & & & & & \\
\hline Shulman et al. [34] & GA (weeks) ${ }^{d}$ & aOR: $0.72^{e}$ & $0.67-0.78$ & BW (per $10 \mathrm{~g})^{\mathrm{b}}$ & aOR: $0.97^{\mathrm{e}}$ & $0.96-0.97$ \\
\hline Tabarez-Carvajal et al. [29] & NR & & & & & \\
\hline Van Sorge et al. [30] & NR & & & & & \\
\hline
\end{tabular}

ROP, retinopathy of prematurity; GA, gestational age; BW, birth weight; NR, not reported; aOR, adjusted odds ratio; RR, relative risk; g, grams. ${ }^{a}$ Association with stage $\geq 3$ ROP for VLBW infants only (BW $<1,500 \mathrm{~g}$ ). ${ }^{\text {b }}$ Multivariate model included antenatal steroids, pre-eclampsia, chorioamnionitis, vaginal delivery, GA, $\mathrm{BW}$, male gender, SGA, resuscitation at birth, respiratory distress syndrome, duration invasive, and noninvasive ventilation, persistent ductus arteriosus requiring treatment, intracranial hemorrhage > grade II, surfactant treatment, total days on oxygen, early- and late-onset sepsis, NEC, bronchopulmonary dysplasia, frequency of red blood cell, breastfeeding more than $80 \%$ of feeding at postnatal 28 days, age of regain BW, relative weight gain at 28 days. ${ }^{c}$ Multivariate model included sex, race, GA, BW, continuous invasive, and noninvasive mechanical ventilation, lack of respiratory support, respiratory distress syndrome, and

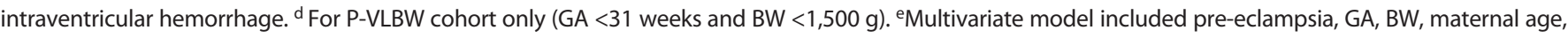
infant sex, multiple gestations, maternal race/ethnicity, alcohol use, and tobacco exposure.

infants born at $\mathrm{GA} \leq 30$ weeks or $\mathrm{BW} \leq 1,500 \mathrm{~g}$, which represent the current screening guidelines in the United States, was $30.7 \%$ (range 27.2 to $34.1 \%$ ) [26, 34].

Nine studies aimed to determine the association between demographic risk factors and the risk of ROP (Table 2). Pooled data showed that risk of any, severe, and treatment-requiring ROP was highest for 23 weeks GA; which was $66.5,40.3$, and $39.4 \%$, respectively (Table 3 ). At 30 weeks, the risk decreased to $4.4,0.4$, and $0.1 \%$, respectively. The threshold at which severe ROP risk was less than $1 \%$ occurred at 29 weeks GA.

Only six studies examined the association between GA and ROP. Of these, all found that a lower GA was significantly associated with a higher risk of ROP. Of the six included studies, only four could be pooled based on a median adjusted OR (aOR). For the four studies pooled, the mean GA ranged from 26.9 to 31.7 weeks. The median aOR for GA by weeks among these studies was 0.7 (range 0.5-0.8) [19, 22, 26, 34]. Thus, for every week decrease in GA, there was a median of 1.4 (range 1.2-1.9) times the odds of developing ROP.

For very low BW (VLBW) (i.e., infants $\leq 1,500 \mathrm{~g}$ ), the risk of any ROP and severe ROP was 29.60 and $9.10 \%$, respectively. For infants with a $\mathrm{BW} \leq 1,000 \mathrm{~g}$, the risk of any, severe, and treatment-requiring ROP was 34.5, 21.7, and $18.4 \%$, respectively. The threshold at which severe ROP risk was less than $1 \%$ occurred at $1,500-2,000 \mathrm{~g}$. 


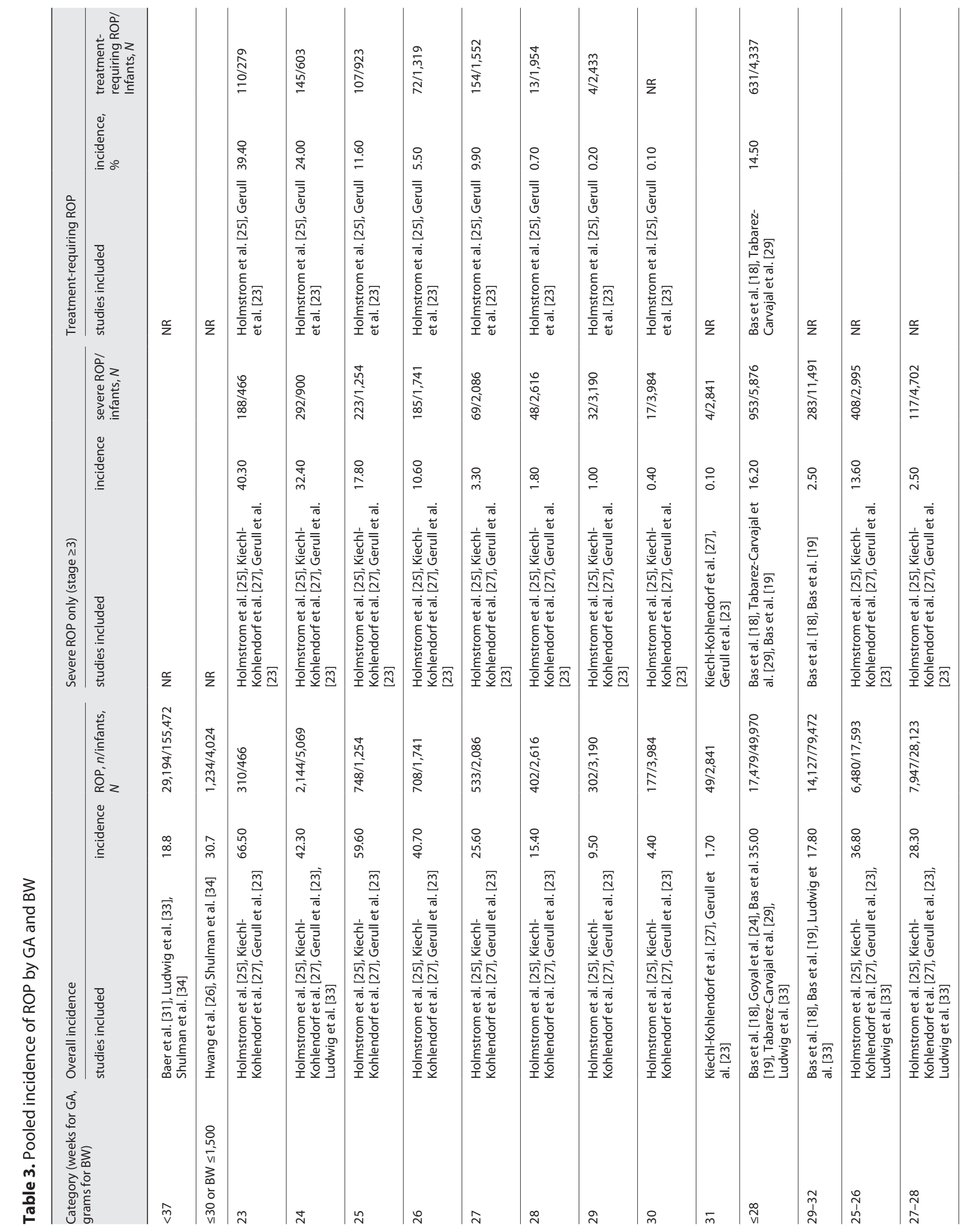




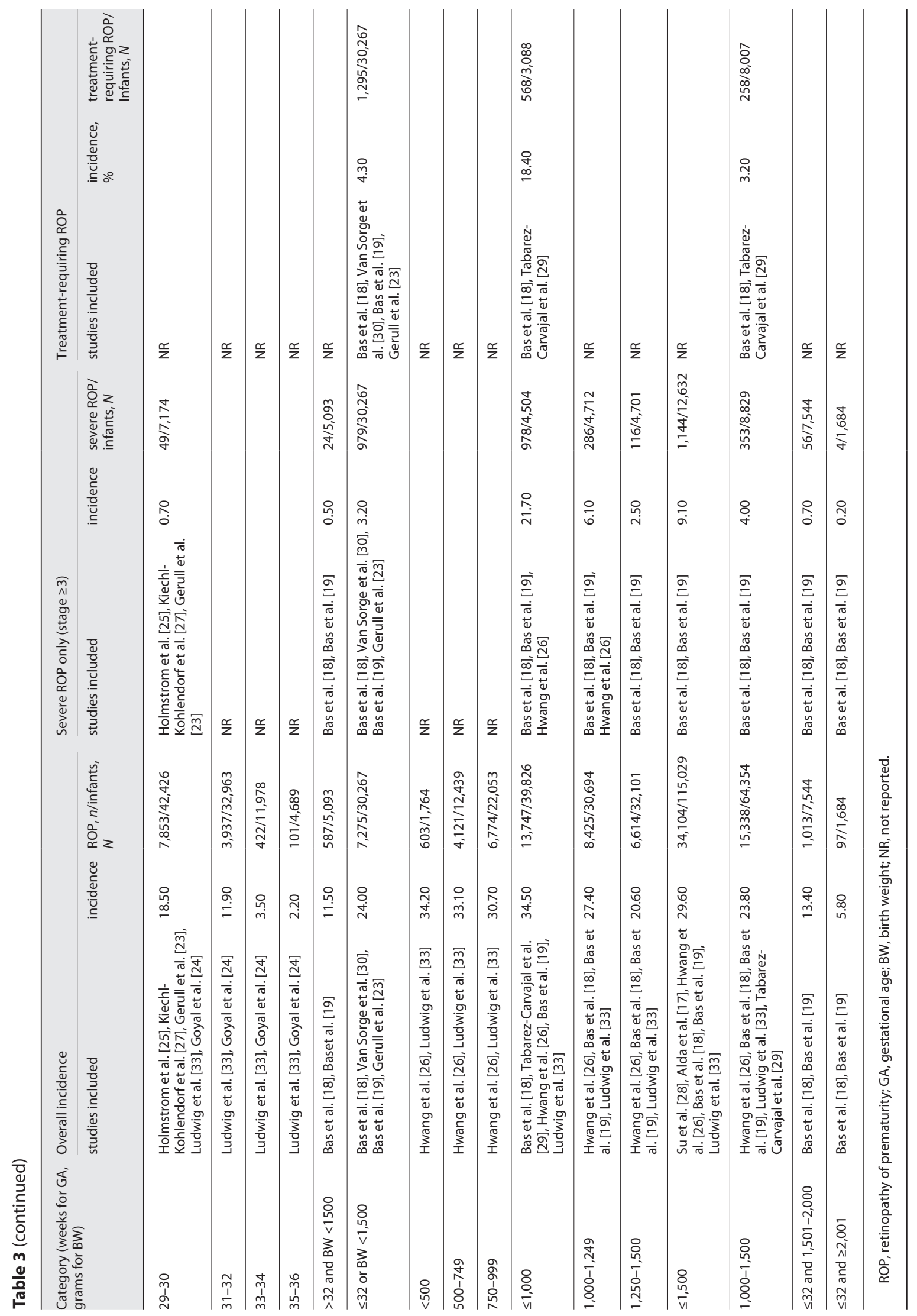


Only five studies examined the association between BW and ROP. Of these, all found that a lower BW was significantly associated with a higher risk of ROP [19, 21, $22,33,34]$. Only two studies could be analyzed to produce a pooled median aOR for BW (mean BW 1,204-1,457 g). For this analysis, the median aOR for BW in grams was 0.994 (range 0.990-0.998) [19, 22]. Per $100 \mathrm{~g}$, the median aOR for BW was 0.5 (range 0.3-0.8). Thus, for every 100 $\mathrm{g}$ decrease in $\mathrm{BW}$, there was a median of 1.8 (range 1.22.7) times the odds of developing ROP. For both GA and BW, one study of VLBW neonates found that they were nonsignificantly associated with higher risk of severe ROP (stage $\geq 3$ ) [26].

\section{Influence of Study Period and Geography on Risk Factors}

Online supplementary Table 7 reports the mean GA and BW of infants with ROP by study period. We did not identify any trends in ROP risk over the prespecified data collection period (i.e., 2010-2020). Studies from geographic region 2 representing higher neonatal mortality were found to have the highest mean GA of infants with all, severe, and treatment-only ROP $(28.5,26.5$, and 28.0 weeks, respectively) when compared to studies from geographic region 1 (26.5, 24.3, and 25.4 weeks, respectively). Geographic region 2 also had the study with the highest mean BW for treatment-only ROP at 1,083 g.

\section{Other Demographic Risk Factors}

The risk of ROP for various demographic risk factors is included in online supplementary Table 8 . No definitive association between sex or ethnicity and risk of ROP was observed $[33,34]$. Some risk factors such as neonatal sepsis $[19,21,22](n=3$, median OR 2.02, range 1.423$2.85)$ and BPD/CLD $[19,21,33](n=3$, median OR 1.94, range 1.70-13.203) were significantly associated with a higher risk of ROP. Others, such as large for GA ( $n=1$, relative risk range $0.4-0.5$ ), were significantly associated with a lower risk of ROP [31]. Small for GA (SGA) was associated with a higher risk of severe ROP and all ROP (GA 28-38 weeks) in two studies [31, 32]. However, SGA was also associated with a lower risk of severe ROP in VLBW infants compared to non-SGA infants in one study [19]. Similarly, SGA infants had a lower risk of ROP compared to non-SGA infants in the youngest GA group (25-27 weeks) only [31]. While blood transfusion was not significantly associated with ROP in three studies $[21,22$, 26], a higher number of blood transfusions was a significant risk factor for ROP on multivariate analysis in two studies $[19,24]$. Pre-eclampsia was associated with a low- er risk of ROP in VLBW infants in two studies $[19,34]$. Other risk factors were reported by one study each and can be found on online supplementary Table 9 .

\section{Discussion}

We performed a systematic review on the epidemiology of patients with ROP in large, population-based studies. The incidence of ROP among preterm infants was $18.8 \%$. We found that among infants born at GA $\leq 30$ weeks or BW $\leq 1,500 \mathrm{~g}$, the risk of ROP was $30.7 \%$. This supports the many screening protocols which set criteria based on high-risk demographic characteristics.

The risk of ROP increases proportionally with lower GA and BW (Fig. 1). Overall, ROP is most common in infants born $<26$ weeks gestation [1]. In our study, a diagnosis of any ROP occurred in two of three infants born at GA 23 weeks and one of three infants born at BW $\leq 1,000 \mathrm{~g}$. This study adds to existing literature by reporting that for each $100 \mathrm{~g}$ of decrease in BW, there was 1.8 times higher odds of developing ROP. For each week of decrease in GA, there was 1.4 times higher odds of developing ROP. While studies have previously suggested that BW is a proxy for GA, BW has been suggested to be independently associated with the risk of ROP in prior studies $[8,35,36]$.

Current ROP screening guidelines have set inclusive BW and GA thresholds to ensure all potential infants requiring treatment are captured. While the current American guidelines have recommended screening of all infants $\leq 30$ weeks of GA or $\leq 1,500$ g of BW [37], refinements to the screening criteria may be emerging to improve the sensitivity of screening. In the recent Postnatal Growth and ROP study, infants were screened if they met criteria that include postnatal weight gain or presence of hydrocephalus [38]. Recent research by Binenbaum et al. [38] showed that the Growth and ROP study criteria had improved sensitivity (100\% compared to $99.6 \%$ for current American guidelines) for type one ROP and resulted in a $30 \%$ reduction in number of infants requiring examination. As screening guidelines evolve to improve sensitivity, studies on the demographic risk factors of ROP are important to ensure that screening guidelines are sensitive to the demographic trends of the population over time.

While the aim of our study was to examine GA and BW as risk factors for ROP, several studies also showed that neonatal comorbidities such as sepsis [19, 21, 22], BPD/CLD $[19,21]$, and an increased number of blood 
transfusions $[19,24]$ were associated with higher risk of ROP. Risk factors such as maternal age were not significantly associated with risk of ROP $[19,34]$. Contradictory results existed for other risk factors such as infant sex, SGA, and intrauterine growth restriction, likely due to the heterogeneity of baseline demographics, such as in GA, BW, or ethnicity, in relation to the development of ROP. It is possible that these risk factors have associations with the development of ROP; however, further research is required. In our analysis, 4 studies used expanded screening criteria that reflect an unstable clinical course (e.g., oxygen exposure, intrauterine growth restriction, and sepsis) to screen infants who are larger and older than the specified GA and BW thresholds. This practice varied between studies and warrants further investigation. Overall, a lower (89\%-95\%) $\mathrm{SpO}_{2}$ level has been found to be associated with a lower risk of treatment-requiring ROP compared to a higher (91-95\%) $\mathrm{SpO}_{2}$ level [39].

The understanding of risk factors is important to facilitate screening, early diagnosis, and treatment of ROP while limiting unnecessary eye examinations. The screening criteria, however, must be tailored for the geographic region. This is emphasized by our finding that regions with higher neonatal mortality rates had the highest mean GA of infants with all, severe, and treatment-only ROP, and the highest mean BW for treatment-only ROP [17, $19,22]$.

There are several important strengths in our study. This study represents the first review that summarizes the risk of any, severe, and treatment-requiring ROP stratified by GA and BW. Second, our review is associated with a high degree of external validity with studies derived from 14 countries. The exclusion of studies published prior to 2010 reduced confounders from changes in neonatal care over the decades. Third, studies had low risk of bias and screened infants at a national level in most cases.

This study should be interpreted in light of the following limitations. First, comparison between studies was difficult due to variability in entry criteria, time period, and methodology. There are important differences in neonatal practices in various regions. In addition, data largely depended on regional screening guidelines, which may not be appropriate in all instances. While a distinction between regions with lower and higher neonatal mortality was made, subgroup analysis could not be conducted due to limited data for each outcome. Second, the associations between the risk of ROP and demographic risk factors were derived via pooled aORs; however, the adjustment made within each study varied. To highlight the uncertainty associated with these estimates, ranges were provided. Third, nonrandomized studies were subject to confounding bias. Fourth, the data on additional risk factors of ROP, such as race, were limited as they were not reported by large population-based studies. Future population-based studies are needed to support our findings for demographic risk factors not captured by our analysis.

\section{Conclusion}

In conclusion, the risk of ROP for infants born at GA $\leq 30$ weeks or $\mathrm{BW} \leq 1,500 \mathrm{~g}$ was $30.7 \%$. For every week decrease in GA, there was 1.4 times the odds of developing ROP. BW was also independently associated with ROP. For every $100 \mathrm{~g}$ decrease in BW, there was 1.8 times the odds of developing ROP. A higher risk of ROP was also found for infants who had neonatal sepsis, BPD/CLD, or a higher number of blood transfusions. Further research is required to clarify the role of additional risk factors. As the survival of preterm infants improve, it is important to monitor the risk factors of ROP in infants and refine their associations with ROP. ROP screening criteria must also be tailored for the geographic region.

\section{Statement of Ethics}

An ethics statement is not applicable because this study is based exclusively on published literature.

\section{Conflict of Interest Statement}

M.M.P. received financial support (to institution) from PSI Foundation. P.J.K. is a member of advisory board in Novartis, Alcon, Bayer, Roche, and Novelty Nobility; received financial support (to institution) from Bayer, Roche, and Novartis; received financial support from Novartis, Bayer; and Equity owner in ArcticDx. R.H.M. is a member of advisory board in Bayer, Novartis, Allergan, and Roche and received financial support (to institution) from Bayer and Novartis. K.M. is a member of advisory board in Santen Inc. and Novartis, and received research funding from Bayer. N.N.T. received nonfinancial support from Bayer.

\section{Funding Sources}

No funding was received. 


\section{Author Contributions}

P.J.K., P.A., R.H.M., K.M., and N.N.T. were involved in the conceptualization/design, methodology, and supervision of the study. C.W.Y., M.M.P., and A.S.D. were involved in the methodology, data curation, investigation, and formal analysis. The original draft was written by C.W.Y. and M.M.P. with reviewing and editing from all authors. Both C.W.Y. and A.S.D. have verified the underlying data.

\section{Data Availability Statement}

All data generated or analyzed during this study are included in this article and its online supplementary material files. Further inquiries can be directed to the corresponding author.

\section{References}

1 Blencowe H, Lawn JE, Vazquez T, Fielder A, Gilbert C. Preterm-associated visual impairment and estimates of retinopathy of prematurity at regional and global levels for 2010 . Pediatr Res. 2013;74(Suppl 1):35-49.

2 Sapieha P, Joyal JS, Rivera JC, KermorvantDuchemin E, Sennlaub F, Hardy P, et al. Retinopathy of prematurity: understanding ischemic retinal vasculopathies at an extreme of life. J Clin Invest. 2010;120:3022-32.

3 Palmer EA, Flynn JT, Hardy RJ, Phelps DL, Phillips CL, Schaffer DB, et al. Incidence and early course of retlnonathy of prematurity. Ophthalmol. 1991;98:1628-40.

4 Isaza G, Donaldson L, Chaudhary V. Increased incidence of retinopathy of prematurity and evolving treatment modalities at a Canadian tertiary centre. Can J Ophthalmol. 2019;54:269-74.

5 Wilson-Costello D, Friedman H, Minich N, Fanaroff AA, Hack M. Improved survival rates with increased neurodevelopmental disability for extremely low birth weight infants in the 1990s. Pediatrics. 2005; 115:997-1003.

6 Fierson WM. Screening examination of premature infants for retinopathy of prematurity. Pediatrics. 2018;142(6):e20183061.

7 Mora JS, Waite C, Gilbert CE, Breidenstein B, Sloper JJ, Mora JS. A worldwide survey of retinopathy of prematurity screening. Br J Ophthalmol. 2018;102:9-13.

8 Schaffer DB, Palmer EA, Plotsky DF, Metz HS, Flynn JT, Tung B, et al. Prognostic factors in the natural course of retinopathy of prematurity. Ophthalmology. 1993;100:230-7.

9 Yang CY, Lien R, Yang PH, Chu SM, Hsu JF, $\mathrm{Fu} \mathrm{RH}$, et al. Analysis of incidence and risk factors of retinopathy of prematurity among very-low-birth-weight infants in North Taiwan. Pediatr Neonatol. 2011;52:321-6.

10 Allegaert K, Casteels I, Cossey V, Devlieger H. Retinopathy of prematurity: any difference in risk factors between a high and low risk population? Eur J Ophthalmol. 2003;13:784-8.

11 Akkoyun I, Oto S, Yilmaz G, Gurakan B, Tarcan A, Anuk D, et al. Risk factors in the development of mild and severe retinopathy of prematurity. J AAPOS. 2006;10:449-53.
12 Yau GS, Lee JW, Tam VT, Liu CC, Chu BC, Yuen CY. Incidence and risk factors for retinopathy of prematurity in extreme low birth weight Chinese infants. Int Ophthalmol. 2015;35:365-73.

13 Estimation TUIG for CM. Mortality rate, neonatal (per 1,000 live births): The World Bank; 2019. Available from: https://data. worldbank.org/indicator/SH.DYN.NMRT Accessed 2021 Jan 1.

14 Hoy D, Brooks P, Woolf A, Blyth F, March L, Bain C, et al. Assessing risk of bias in prevalence studies: modification of an existing tool and evidence of interrater agreement. J Clin Epidemiol. 2012;65:934-9.

15 The Cochrane Collaboration. GRADEpro GDT; 2017.

16 Guyatt GH, Oxman AD, Vist GE, Kunz R, Falck-Ytter Y, Alonso-Coello P, et al. GRADE: an emerging consensus on rating quality of evidence and strength of recommendations. Br Med J. 2008;336:924-6.

17 Alda E, Lomuto CC, Benítez AM, Bouzas L, Brussa M, Cattaino A, et al. Results of the national program for the prevention of blindness in childhood by retinopathy of prematurity in Argentina (2004-2016). Arch Argent Pediatr. 2018;116:386-93.

18 Bas AY, Koc E, Dilmen U, Oguz SS, Ovali F, Demirel N, et al. Incidence and severity of retinopathy of prematurity in Turkey. Br J Ophthalmol. 2015;99:1311-4.

19 Bas AY, Demirel N, Koc E, Ulubas Isik Di, Hirfanoglu IM, Tunc T. Incidence, risk factors and severity of retinopathy of prematurity in Turkey (TR-ROP study): a prospective, multicentre study in 69 neonatal intensive care units. Br J Ophthalmol. 2018;102:1711-6.

20 Boland RA, Davis PG, Dawson JA, Doyle LW. Outcomes of infants born at 22-27 weeks' gestation in Victoria according to outborn/ inborn birth status. Arch Dis Child Fetal Neonatal Ed. 2017;102:F153-61.

21 Borroni C, Carlevaro C, Morzenti S, De Ponti E, Bozzetti V, Console V, et al. Survey on retinopathy of prematurity (ROP) in Italy. Ital J Pediatr. 2013;39:43.

22 Feghhi M, Altayeb SMH, Haghi F, Kasiri A, Farahi F, Dehdashtyan M, et al. Incidence of retinopathy of prematurity and risk factors in the South-Western Region of Iran. Middle East Afr J Ophthalmol. 2012;19:101-6.
23 Gerull R, Brauer V, Bassler D, Laubscher B, Pfister RE, Nelle M, et al. Prediction of ROP treatment and evaluation of screening criteria in VLBW infants: a population based analysis. Pediatr Res. 2018;84:632-8.

24 Goyal A, Giridhar A, Gopalakrishnan M, Thachil T. Neonatal intensive care unit-based screening program for retinopathy of prematurity and its treatment in an Indian population. Indian J Ophthalmol. 2019;67:828-33.

25 Holmström G, Hellström A, Gränse L, Saric M, Sunnqvist B, Wallin A, et al. New modifications of Swedish ROP guidelines based on 10 -year data from the SWEDROP register. $\mathrm{Br}$ J Ophthalmol. 2020;104:943-9.

26 Hwang JH, Lee EH, Kim EA. Retinopathy of prematurity among very-low-birth-weight infants in Korea: incidence, treatment, and risk factors. J Korean Med Sci. 2015;30(Suppl 1):S88-94.

27 Kiechl-Kohlendorfer U, Simma B, Urlesberger B, Maurer-Fellbaum U, Wald M, Wald M, et al. Low mortality and short-term morbidity in very preterm infants in Austria 2011-2016. Int J Paediatr. 2019;108:1419-26.

28 Su YY, Wang SH, Chou HC, Chen CY, Hsieh WS, Tsao PN, et al. Morbidity and mortality of very low birth weight infants in Taiwanchanges in 15 years: a population based study. J Formosan Med Assoc. 2016;115:1039-45.

29 Tabarez-Carvajal AC, Montes-Cantillo M, Unkrich KH, Trivedi RH, Peterseim MMW. Retinopathy of prematurity: screening and treatment in Costa Rica. Br J Ophthalmol. 2017;101:1709-13.

30 Van Sorge AJ, Termote JUM, Simonsz HJ, Kerkhoff FT, Van Rijn LJ, Lemmens WA, et al. Outcome and quality of screening in a nationwide survey on retinopathy of prematurity in the Netherlands. Br J Ophthalmol. 2014;98:1056-60.

31 Baer RJ, Rogers EE, Partridge JC, Anderson JG, Morris M, Kuppermann M, et al. Population-based risks of mortality and preterm morbidity by gestational age and birth weight. J Perinatol. 2016;36:1008-13.

32 Boghossian NS, Geraci M, Edwards EM, Horbar JD. Morbidity and mortality in small for gestational age infants at 22 to 29 weeks' gestation. Pediatrics. 2018;141(2):141. 
33 Ludwig CA, Chen TA, Hernandez-Boussard T, Moshfeghi AA, Moshfeghi DM. The epidemiology of retinopathy of prematurity in the United States. Ophthalmic Surg Lasers Imaging Retina. 2017;48:553-62.

34 Shulman JP, Weng C, Wilkes J, Greene T, Hartnett ME. Association of maternal preeclampsia with infant risk of premature birth and retinopathy of prematurity. JAMA Ophthalmol. 2017;135:947-53.
35 Fortes Filho JB, Eckert GU, Procianoy L, Barros CK, Procianoy RS. Incidence and risk factors for retinopathy of prematurity in very low and in extremely low birth weight infants in a unit-based approach in southern Brazil. Eye. 2009;23:25-30.

36 Friling R, Axer-Siegel R, Hersocovici Z, Weinberger D, Sirota L, Snir M. Retinopathy of prematurity in assisted versus natural conception and singleton versus multiple births. Ophthalmology. 2007;114:321-4.

37 Fierson WM. Screening examination of premature infants for retinopathy of prematurity. Pediatrics. 2013;131:189-95.
38 Binenbaum G, Tomlinson LA, De Alba Campomanes AG, Bell EF, Donohue P, Morrison $\mathrm{D}$, et al. Validation of the postnatal growth and retinopathy of prematurity screening criteria. JAMA Ophthalmol. 2020;138:31-7.

39 Askie LM, Darlow BA, Finer N, Schmidt B, Stenson B, Tarnow-Mordi W, et al. Association between oxygen saturation targeting and death or disability in extremely preterm infants in the neonatal oxygenation prospective meta-analysis collaboration. J Am Med Assoc. 2018;319:2190-201. 